\title{
Smart Glasses Implementation in Hospitals
}

\author{
XiaoXue Gong, UCSI University, Malaysia \\ Ann Hung Wong, UCSI University, Malaysia \\ (iD https://orcid.org/0000-0003-1679-1057 \\ Rashidova Kamilla Darobovna, UCSI University, Malaysia \\ P. S. JosephNg, UCSI University, Malaysia
}

\begin{abstract}
Visualizing information onto the lens, one can collect data over a wireless network and use a program to convert it into text or images to be transmitted to the display using smart glasses. These products will serve the medical industry. Therefore, the feasibility of using smart glasses in surgery was studied in this study. A technology acceptance model was used to analyze people's acceptance of the use of smart glasses in the medical system. Mix mode analysis of the data was conducted through the explanatory study and the cross-sectional study, and relevant data were collected using an online questionnaire. The study found that surgeons using smart glasses could improve the success rate of surgery.
\end{abstract}

\section{KEYWORDS}

Augmented Reality, Smart Glasses, Surgery, Visualization, Wearable Device

\section{INTRODUCTION}

\section{Background of the Industry}

Smart glasses are a new intelligent product and have a huge development prospect in the next few years. Smart glasses refer to the general name of eyewear devices that have an independent operating system like smartphones and can realize various functions through software installation (Danielsson et al., 2020). It is a new type of wearable intelligent device which has been put forward in recent years and has a good development prospect. It is simple to use, small in size and portable to carry. It is regarded as a high-tech product of the future. Wearable technology has entered the medical field and will change surgery dramatically (Hiranaka et al., 2017). Realize the control mode of hands liberation, use voice, gesture, glasses to control the smart glasses, simplify the operating system (InfoLinker, 2021).

The Coronavirus disease 2019 (COVID-19) was declared a pandemic by the World Health Organization (WHO) on 11th March 2020 (Cucinotta et al., 2020). Close distance will easily get infected in this Covid-19 as the droplets can aerosolize up to 4metres. Especially the healthcare workers will be at a higher risk of occupational transmission of Covid-19 (Guo et al.,2020). Recommendations are in place to limit medical personnel attending to the Covid-19 patient as a protective measure and to conserve personal protective equipment (Ranney et al., 2020). Wearing smart glasses can avoid close contact and minimize sickness for doctor and patients. Thus, a safer practical option will be to 
H1: Cost effective can be conducted for surgical operation in a hospital using smart glass.

$\mathrm{H} 2$ : Surgical operation can be improved in a hospital using smart glasses.

H3: Surqical operation confidence can be enhanced in a hospital using smart

facilitate the virtual presence of key medical personnel in the ICU. On top of that, smart glasses offer a real-time and contemporary solution to mitigate the shortcomings and bridge the communication gap during the COVID-19 pandemic. Besides that, patients also being reviewed and steps in the examination, consultation and documentation processes were developed and compiled into a Standard Operating Procedure (SOP) manual (InfoLinker, 2021). By using Smart glasses, the specialist was able to holistically and continuously "see" the exact first-hand clinical information in real-time and in a "bedside" manner to advise and guide the patient confidently and accurately (Daggubati et al., 2020).

Through the research and analysis of relevant literature, we put forward research questions about the cost, effective and brand of smart glasses according to the viewpoints and methods of the article. According to these questions to carry out research. Through the investigation of these three aspects, it is confirmed that smart glasses can help surgeons to complete operations well and reduce the risk of surgery. Give patients a good operation experience, reduce the wound surface.

According to the above two tables, we put forward the hypothesis (Table 1) of this study. We will analyze the questionnaire and the existing relevant data. To test the operability of the verification of these hypotheses. Whether smart glasses can effectively improve and help surgeons perform operations. By using smart glasses can reduce the time visit, travel distance and cost by participants. These glasses however have been successfully utilised in business and industry to improve safety, productivity, especially in warehouse inventory management, high-risk jobs that required safeguarding while the junior workers required from a distance (Muensterer et al., 2016).

\section{LITERATURE REVIEW}

The literature review shows different points of the processes and further discoveries as well as pros and cons of the implementation of Smart Glasses in different field of the Medical Industry, moreover for the special uses such as preventing accidents during the drowsiness as an extra understanding and features to discover. Smart glasses are small computers that comprise a head-mounted monitor and video camera and can be connected to the internet (Hiranaka et al., 2017). It can be used for remote observation of surgeries and sickness by video streaming. Currently, there are few types of smart glasses in the world. The most common smart glasses for medical purposes are Google Glass. while InfoLinker mainly for surgical video streaming is now commercially available in Japan for industrial purposes which has more than 2 years (Hiranaka et al., 2017). The smart glass feature can be used by remote surgeons for surgical observation. In different forms of operations, such as pediatric surgery, dermatological surgery, and plastic surgery, some surgeons have tried to use Google Glass (Kantor J. 2016). Google Glass, however, was not widely viable until now. Google Glass was not commercially available up to now. Therefore, it is not available to most surgeons for general use. InfoLinker glasses, which are explored in the research, are innovative smart glasses with great potential. It was created for industrial applications, such as task navigation, remote manufacturing, and platforms for sharing know-how (Hiranaka et al., 2017). In this case, there is a comparison between Google Glass and newly explored InfoLinker that should be used more in the medical industry. Since many AR smart glasses are available at the moment, visual augmentation in terms of visual contexts and gesture interaction must be made more realistic and intuitive (Hiranaka et al., 2017). Nevertheless, there has been no study work on exploring this problem in detail. User interactions based on visual contexts 
and gesture interactions were categorized and analyzed using AR smart glasses in this study (Kim, Choi, Park, \& Lee, 2019). Moreover, AR smart glasses are starting to get to be used more because of efficiency and each time updated, as it goes through the stages of detecting so-called problems and upgrading till it stands out in its usage.

In various fields, such as surgery, accessing electronic health records, remote instructions, and education, smart glasses are a device proposed to support health care professionals (Romare, C. and Skär, L., 2020). They are a computer interface worn as a pair of glasses, which, via a prism, displays information within the field of view of the user. Increased awareness is given about various scenarios in which health care professionals in clinical practice in anaesthesia departments and ICUs could use smart glasses (Hiranaka et al., 2017). In the images shown on computer screens, various medical procedures are performed. The operator needs to take his/her eyes off the activity field in most such processes to see the display. Additionally, for example, to fluoroscopy, images on displays include vital sign display, endoscope, and computer navigation, as well as traditional images such as X-ray images, computed tomography, and magnetic resonance imaging. Using the PicoLinker helps the operator to hold his/her eyes on the field of operation when viewing the photos (Hiranaka et al., 2017). Besides, several earlier studies have been using heavy computer-based systems by M. I. ChaconMurguia, and C. Prieto-Resendiz and A. Koesdwiady, R. Soua, F. Karray, and M. S. Kamel, which are inadequate or unsatisfactory for real-world customers (Kim, Choi, Park, and Lee, 2019, CM Kang et. al 2015). Besides, DFD systems based on image/video recognition are not suitable (Wang, Ong, and Nee, 2016). Due to inadequate illumination sources for night driving (Munusamy et al., 2021).

\section{METHODOLOGY}

\section{To Investigate the Acceptance of Smart Glasses Surgery Using Several Research}

\section{Quantitative Research}

Quantitative research is the numerical representation and manipulation of observation to investigate the phenomena by gathering and collecting numerical data that are analyzed using mathematically based methods (JosephNg et al., 2021-2011, Maruyama et al., 2018). We will use TAM to consider the acceptance of doctors and patients for smart glasses surgery and the impact of smart glasses on surgery. This is mainly because we will use a closed questionnaire to collect data. Quantitative analysis itself is the use of mathematical-statistical methods to analyze data. Analyze the data to see if the explanation is valid (Figure 1).

\section{Target Population}

The target population is the special population suitable for the investigation and research. According to the product and service objects of the experiment, the subjects of this study are patients and surgeons in Malaysian hospitals.

\section{Sample Size}

In qualitative research, the sample size should meet the needs of the research. The sample size should not be too large nor too small. Sampling too small can not guarantee the comprehensiveness and accuracy of the data, and there is a large error. On the contrary, if the sample size is too large, more energy and time will be needed to conduct data analysis, otherwise, it is impossible to conduct a detailed analysis of all the data. Therefore, this study plans to collect 120 survey respondent.

\section{Measurement}

We prepared a self-administered questionnaire, which summarized some single choice questions that were easy for respondents to answer according to relevant reference materials. It is easy for the surveyor to understand and answer. As Malaysia is a multilingual country, this questionnaire will be written in 
Figure 1. Research model

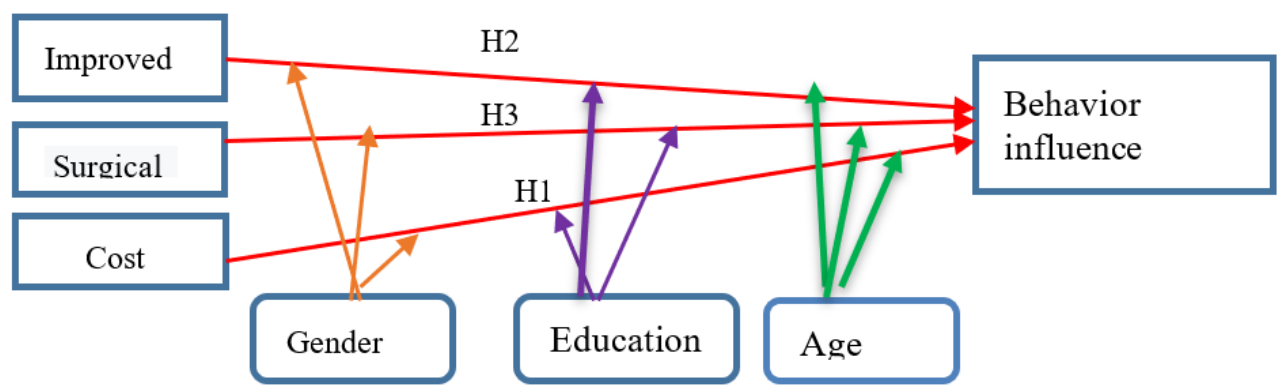

English, the common language. To facilitate interviewees' language and cultural exchanges. Likert scale questionnaire was used to collect data, which was used to measure the intensity of the extent to which the surveyors agreed or disagreed with a certain question. Strong to weak to strong. There are 5 of them including strongly disagree (1), disagree (2), undecided (3), agree (4) to strongly agree (5).

\section{Data Collection}

Due to the influence of the CoviD-19 epidemic, this study mainly collected questionnaire data through the online questionnaire. In this study, primary data were used for data analysis. The survey questions were based on usage questions and references. Each investigator can only conduct one survey, and no repetition or modification is allowed. Target groups were invited to conduct questionnaires through the Internet, mainly through WhatsApp, Facebook and WeChat. Besides, some target groups were interviewed and teams and zoom were applied. SPSS (Statistical Package for Social Sciences) Version 20 was used for the data analysis software of this study. Using SPSS can effectively verify the accuracy of experimental data. At the same time, the hypothesis can be tested by using the multiple linear regression method and the correct conclusion can be drawn.

As can be seen from Table 4, the results of CA Values are all greater than 0.8, indicating high credibility of cost, improved surgery and surgical confidence and behaviour influence, and D-g Rho Values indicate high internal consistency among variables.CR indicates a high composite credit rating of the variable. At the same time AVE\&gt; 0.5 , indicating that the true data in the experimental data is greater than the error data. The experimental data collection is real and effective, and there is an interactive relationship.

\section{Findings}

Through this investigation, we have concluded that smart glasses can help surgeons better complete operations. Patients will also be able to receive a better vision from surgeons who wear smart glasses during surgery, contributing to the success of the operation.I

\section{Table 2. Reliability and Validity}

\begin{tabular}{|c|c|c|c|c|c|}
\hline Variables & No. Items & $\mathrm{CA}$ & DG rho & $\mathrm{CR}$ & AVE \\
\hline Cost & 6 & 0.911 & 0.916 & 0.932 & 0.697 \\
\hline Improved surgery & 7 & 0.884 & 0.897 & 0.910 & 0.592 \\
\hline Surgical Confidence & 7 & 0.897 & 0.904 & 0.919 & 0.618 \\
\hline Behavior influence & 6 & 0.850 & 0.856 & 0.888 & 0.571 \\
\hline
\end{tabular}

Note: CA: Cronbach's Alpha; DG rho - Dillon-Goldstein's rho; CR - Composite Reliability; AVE - Average Variance Extracted 

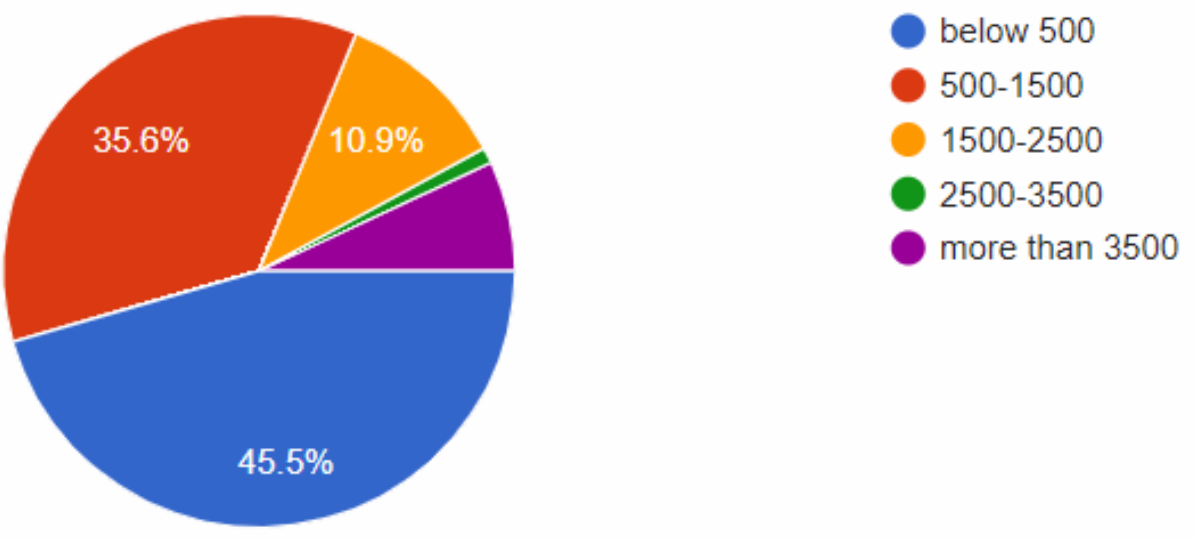

\section{Improve Surgery}

In general, the quality of the image comes from the number of pixels on the camera and the speed of the network. For the current network, we usually have 4G or 5G. It's relatively stable. But it's also when there's a poor signal from the WiFi network. This is to strengthen the operating room network signal, to ensure the smooth completion of the operation. For the pixel size of the camera, a zoomable HD camera is used, while smart glasses can be connected to endoscopes and microscopes. To ensure that the image can be displayed on the monitor.

\section{Cost}

In terms of price, $45.4 \%$ of people hope that the price of smart glasses is lower than 500RM. 35.6\% of people are willing to accept the smart glasses of 500RM to 1500RM. Based on the survey results, the price of smart glasses should not be higher than 1500RM so that more consumers can buy them (Figure 2).

\section{Confidence}

When surgeons use smart glasses for surgery, they can improve the accuracy of the operation, avoid head movement during the operation, and reduce the operation time (Chang, Chen, and Chiou, 2018). Improve the success rate of operation. For patients, there will be fewer doctor mistakes during surgery, less time spent during surgery and less use of anaesthetics. Avoid allergy and adverse reactions caused by large amounts of anaesthetics. Increase your confidence in the success of the operation.

\section{DISCUSSION}

Based on the results of the experimental study, we discussed the research and development, production and sales of our company's smart glasses.

\section{Product Technology}

According to the survey, people attach more importance to speech manipulation and eye movement manipulation. Install HD camera and motion capture camera. Realize high-definition display, 3D imaging, precision measurement, etc. AR technology is mainly used for virtual surgery. Virtual surgery 
Table 3. Competitor product comparison

\begin{tabular}{|c|c|c|c|}
\hline $\mathrm{Nb}$ & Product & Application & Purpose \\
\hline 1 & Smart glasses & $\begin{array}{l}\text { For remote consultation and ward } \\
\text { rounds }\end{array}$ & $\begin{array}{l}\text { Help remote and } \\
\text { poor people to see a } \\
\text { doctor to facilitate } \\
\text { doctors to understand } \\
\text { and record the pa- } \\
\text { tient's condition. }\end{array}$ \\
\hline 2 & $\begin{array}{cc}\mathrm{HD} & \text { smart } \\
\text { glasses } & \end{array}$ & $\begin{array}{l}\text { It is mainly used for ICU ward } \\
\text { monitoring, monitoring ICU patients } \\
\text { through infrared camera, and trans- } \\
\text { mitting data to medical staff wearing } \\
\text { smart glasses. Se they can monitor } \\
\text { patients in real time }\end{array}$ & $\begin{array}{l}\text { Reduce the cross } \\
\text { infection of medical } \\
\text { staff, reduce the work- } \\
\text { load of medical staff. }\end{array}$ \\
\hline 3 & $\begin{array}{l}\text { Ultra } \\
\text { smart glasses }\end{array}$ & $\begin{array}{l}\text { Mainly used in surgery, surgeons } \\
\text { can use the glasses to clearly see le- } \\
\text { sions and measure their size. Blood } \\
\text { flow and blood pressure can also be } \\
\text { measured. }\end{array}$ & $\begin{array}{l}\text { Reduce the risk of } \\
\text { surgery and improve } \\
\text { the success rate of sur- } \\
\text { gery. }\end{array}$ \\
\hline
\end{tabular}

is virtual imaging of reality. 3D imaging of surgery through a virtual system makes the surgery more real. It is mainly used for medical teaching, and the simulation of the early stage of operation, to realize the pretreatment of the problems in the operation. Reduce errors and errors during surgery.

\section{Cost-Effective}

Our products mainly serve the medical industry, and we need to design different products according to different uses, to customize different prices.

Meet the needs of more consumers. The details are shown in Table 3:

\section{Advertising}

Advertising is an important way to enhance the brand effect. The finished product is produced for sale, but as a new product, how to better achieve product publicity. It's very important to get the product into the market. Usually, the quality of the product is one thing, but the advertising effect has an important effect on the product. Especially the cost of advertising. E-commerce advertising costs are generally lower than television advertising costs. We are willing to keep the cost of advertising within $15 \%$ of the cost of the company's products, above which a negative return may occur. Therefore, the promotion of advertisements should be carried out on the Internet as much as possible or entirely through sales platforms, cloud services, mobile phone APPs, etc. The initial delivery volume should be more when customers can accept the product and the increase in purchasing power. To control the amount of advertising, advertising will be centralized delivery. Achieve the goal of reducing advertising cost. People's desire to buy will often be bought by the brand reputation and follow the trend, so we should pay special attention to the design of digital network advertising, publicity.

\section{CONCLUSION}

In terms of smart glasses' globalization, the application of smart glasses in various fields is still in its infancy, requiring more study and exploration. To improve the use of smart glasses in a variety of complex environments, reasonable use and coordination of language recognition technology, image 
recognition technology, augmented reality technology, and other technologies are required (Jayasudha and Kabadi, 2020). The limitations of the study are linked to the times of the Covid -19 where more exploration is required for future research and widen the research scope and accessibility. As a result of our research, we have concluded that smart glasses can assist surgeons in completing operations more efficiently. Patients will also benefit from surgeons wearing smart glasses during surgery, which will improve their vision and contribute to the operation's success. The product's position and awareness are both extremely important. Once the product passes the required norms and efficiency/ effectiveness it will bring the process of the medical industry and personal usage to the next level. 


\section{REFERENCES}

Chang, W., Chen, L., \& Chiou, Y. (2018). Design and Implementation of a Drowsiness-Fatigue-Detection System Based on Wearable Smart Glasses to Increase Road Safety. IEEE Transactions on Consumer Electronics, 64(4), 461-469. doi:10.1109/TCE.2018.2872162

Cucinotta, D., \& Vanelli, M. (2020). WHO declares COVID-19 a pandemic. Acta Biomedica, 91(1), 157-160. PMID:32191675

Daggubati, L. C., Eichberg, D. G., Ivan, M. E., Hanft, S., Mansouri, A., Komotar, R. J., D’Amico, R. S., \& Zacharia, B. E. (2020). Telemedicine for Outpatient Neurosurgical Oncology Care: Lessons Learned for the Future During the COVID-19 Pandemic. World Neurosurgery, 139, e859-e863. doi:10.1016/j.wneu.2020.05.140 PMID:32450309

Danielsson, O., Holm, M., \& Syberfeldt, A. (2020). Augmented reality smart glasses in industrial assembly: Current status and future challenges. Journal of Industrial Information Integration, 20, 100175. doi:10.1016/j. jii.2020.100175

Guo, Z. D., Wang, Z. Y., Zhang, S. F., Li, X., Li, L., Li, C., Cui, Y., Fu, R.-B., Dong, Y.-Z., Chi, X.-Y., Zhang, M.Y., Liu, K., Cao, C., Liu, B., Zhang, K., Gao, Y.-W., Lu, B., \& Chen, W. (2020). Aerosol and Surface Distribution of Severe Acute Respiratory Syndrome Coronavirus 2 in Hospital Wards, Wuhan, China. Emerging Infectious Diseases, 26(7), 1583-1591. doi:10.3201/eid2607.200885 PMID:32275497

Hiranaka, T., Fujishiro, T., Hida, Y., Shibata, Y., Tsubosaka, M., Nakanishi, Y., Okimura, K., \& Uemoto, H. (2017). Augmented reality: The use of the PicoLinker smart glasses improves wire insertion under fluoroscopy. World Journal of Orthopedics, 8(12), 891-894. doi:10.5312/wjo.v8.i12.891 PMID:29312847

Hiranaka, T., Nakanishi, Y., Fujishiro, T., Hida, Y., Tsubosaka, M., Shibata, Y., Okimura, K., \& Uemoto, H. (2017). The Use of Smart Glasses for Surgical Video Streaming. Surgical Innovation, 24(2), 151-154. doi:10.1177/1553350616685431 PMID:28068887

Jayasudha, K., \& Kabadi, M. G. (2020). Soft tissues deformation and removal simulation modelling for virtual surgery. International Journal of Intelligence and Sustainable Computing, 1(1), 83-100. doi:10.1504/ IJISC.2020.104830

JosephNg, P.S., Kang, C.M., Choo, P.Y., Wong, S.W., Phan, K.Y., \& Lim, E.H. (2015). Beyond cloud infrastructure services in medium-size manufacturing, International symposium on mathematical sciences \& computing research, IEEE Explore, 150-155.

JosephNg, Moy, Mahmood, Wan, Yuen, Hui, \& Theam (2016). EaaS: Available yet Hidden Infrastructure inside MSE. 5th International Conference on Network, Communication, and Computing, ACM International Conference Proceeding Series, 17-20.

JosephNg, P.S., Kang, C.M., Mahmood, A.K., Choo, P.Y., Wong, S.W., Phan, K.Y., \& Lim, E.H. (2016). Exostructure Services for Infrastructure Resources Optimization. Journal of Telecommunication, Electronic \& Computer Engineering, 8(4), 65-69.

JosephNg, P.S. (2018). EaaS Optimization: Available yet hidden information technology infrastructure inside medium-size enterprises. Journal of Technological Forecasting and Social Change, 132(July), 165-173.

JosephNg, P.S. (2019). EaaS Infrastructure Disruptor for MSE. International Journal of Business Information Systems, 30(3), 373-385.

JosephNg, P.S., Loh, Y.F., \& Eaw, H.C. (2020). Grid Computing for MSE during Volatile Economy. International Conference on Control, Automation and Systems, IEEE Explore, 709-714.

JosephNg, P.S. \& Eaw, H.C. (2021), Making financial sense from EaaS for MSE during economic uncertainty. Advances in Intelligent Systems and Computing.

JosephNg, P.S. \& Eaw, H.C. (2021). Making financial sense from EaaS for MSE during economic uncertainty. Advances in Intelligent Systems and Computing.

JosephNg, P.S. \& Eaw, H.C. (2021). Still Technology Acceptance Model? Reborn: Exostructure as a Service Model. International Journal of Business Information Systems. 
JosephNg, P. S., Mahmood, A. K., Choo, P. Y., Wong, S. W., Phan, K. Y., \& Lim, E. H. (2014). IaaS Cloud Optimization during Economic Turbulence for Malaysia Small and Medium Enterprise. International Journal of Business Information Systems, 16(2), 196-208.

JosephNg, P. S., Mahmood, A. K., Choo, P. Y., Wong, S. W., Phan, K. Y., \& Lim, E. H. (2015). Barebone cloud IaaS: Revitalization disruptive technology. International Journal of Business Information Systems, 18(1), 107-126.

JosephNg \& Kang. (2016). Beyond barebone cloud infrastructure services: Stumbling competitiveness during economic turbulence. Journal of Science and Technology, 24(1), 101-121.

Kang, C. M. (2015), A study on integrating penetration testing into the information security framework for Malaysian higher education institutions. IEEE International Symposium on Mathematical Sciences and Computing Research, 156-161.

Kantor, J. (2016). First look at Google Glass in dermatology, Mohs surgery, and surgical reconstruction. JAMA Dermatology, 150(11), 1191. doi:10.1001/jamadermatol.2014.1558 PMID:25389790

Kim, M., Choi, S., Park, K., \& Lee, J. (2019). User Interactions for Augmented Reality Smart Glasses: A Comparative Evaluation of Visual Contexts and Interaction Gestures. Applied Sciences (Basel, Switzerland), 9(15), 3171. doi:10.3390/app9153171

Levin, K. A. (2016). Study design III: Cross-sectional studies. Evidence-Based Dentistry, 7(1), 24-25. doi:10.1038/sj.ebd.6400375 PMID:16557257

Maruyama, K., Watanabe, E., Kin, T. K., Kumakiri, A., Noguchi, A., Nagane, M., \& Shiokawa, Y. (2018). Smart Glasses for Neurosurgical Navigation by Augmented Reality. Operative Neurosurgery, 15(5), 551-556.

Muensterer, O. J., Lacher, M., \& Zoeller, C. (2016). Google Glass in pediatric surgery: An exploratory study. International Journal of Surgery, 12, 281-289.

Munusamy, T., Karuppiah, R., Bahuri, N. F. A., Sockalingam, S., Cham, C. Y., \& Waran, V. (2021). Telemedicine via Smart Glasses in Critical Care of the Neurosurgical Patient. World Neurosurgery, 145(1), 53-60. doi:10.1016/j. wneu.2020.09.076 PMID:32956888

JosephNg, Choo, Wong, Phan, \& Lim. (2012). Malaysia SME ICT During Economic Turbulence. International Conference on Information \& Computer Network, 67-71.

JosephNg, P. S., Yin, C. P., Wan, W. S., \& Nazmudeen, M. S. H. (2011), Energizing ICT Infrastructure for Malaysia SME during Economic Turbulence, Student Conference on Research and Development, IEEE Explore, 328-322.

Ranney, M.L., Griffeth, V., \& Jha, A.K. (2020). Critical supply shortages - The need for ventilators and personal protective equipment during the Covid-19 pandemic. N Engl J Med., 382(18).

Romare, C., \& Skär, L. (2020). Smart Glasses for Caring Situations in Complex Care Environments. JMIR mHealth and uHealth, 8(4), e16055.

Wang, X., Ong, S. K., \& Nee, A. Y. C. (2016). Real-virtual components interaction for assembly simulation and planning. Robotics and Computer-integrated Manufacturing, 2016(41), 102-114.

Gong XiaoXue is a post graduate student at UCSI University.

Wong Ann Hung is a post graduate student at UCSI University.

Kamilla Rashidova Darobovna is a post graduate student at UCSI University.

JosephNg Poh Soon graduated with PhD (IT), Master in Information Technology (Aus), Master in Business Administration (Aus), and Associate Charted Secretary (UK) with various instructor qualifications, professional certifications and industry memberships. He is currently the Head of Praxis, Industry and Community Engagement (PICE). With his blended technocrat mix of both business senses and technical skills, has held many multinational corporation senior management positions, global posting and leads numerous $24 \times 7 \mathrm{global}$ mission-critical systems. A humble young manager nominee twice, five teaching excellence awards recipient, numerous research grants and hundreds of citations. He has appeared in LIVE television prime time CyberSecurity talk show and overseas teaching exposure. His current researches are on strategic IT infrastructure optimization and digital transformation. 